\title{
Study on Thixojoining Process Using Partial Remelting Method
}

\author{
M. N. Mohammed, M. Z. Omar, M. S. Salleh, and K. S. Alhawari \\ Department of Mechanical and Materials Engineering, Faculty of Engineering and Built Environment, Universiti Kebangsaan Malaysia, \\ 43600 Selangor, Malaysia
}

Correspondence should be addressed to M. Z. Omar; zaidi5886@gmail.com

Received 31 January 2013; Revised 16 April 2013; Accepted 22 April 2013

Academic Editor: Rui Vilar

Copyright ( $) 2013$ M. N. Mohammed et al. This is an open access article distributed under the Creative Commons Attribution License, which permits unrestricted use, distribution, and reproduction in any medium, provided the original work is properly cited.

Cold-work tool steel is considered to be a nonweldable metal due to its high percentage content of carbon and alloy elements. The application of a new process of the semisolid joining of two dissimilar metals is proposed. AISI D2 cold-work tool steel was thixojoined to 304 stainless steel by using a partial remelting method. After thixojoining, microstructural examination including metallographic analysis, energy dispersive spectroscopy (EDS), and Vickers hardness tests was performed. From the results, metallographic analyses along the joint interface between semisolid AISI D2 and stainless steel showed a smooth transition from one to another and neither oxides nor microcracking was observed. Hardness values obtained from the points in the diffusion zone were much higher than those in the 304 stainless steel but lower than those in the AISI D2 tool steel. The study revealed that a new type of nonequilibrium diffusion interfacial structure was constructed at the interface of the two different types of steel. The current work successfully confirmed that avoidance of a dendritic microstructure in the semisolid joined zone and high bonding quality components can be achieved without the need for force or complex equipment when compared to conventional welding processes.

\section{Introduction}

Cold-work tool steel has many attractive properties such as high wear resistance, high compressive strength, high hardness after hardening, and good dimension stability during heat treatment. Due to the growing demand for cold-work tool steel in various industrial applications, it is crucial to improve the welding technique for joining cold-work tool steel. However, welding this type of material is very difficult and it is almost impossible to achieve a good result using conventional processes. There have been a number of reported studies on using a feedstock material with a thixotropic characteristic (globular microstructure) to weld by employing different methods such as laser welding and friction stir welding, but problems arise with these methods including porosities, loss of alloying elements, bad geometry, and softening of the heat-affected zone resulting in a nonhomogenous microstructure in the heat-affected zone caused by the remelting temperature [1-3]. Clearly this last effect is particularly undesirable from a metallurgic point of view due to the lack of control of the microstructure. Moreover, the high temperatures which are necessary to achieve the complete melting of the various parts of the substrate induce a heat-affected zone. In this zone, deterioration of the fracture toughness, corrosion resistance, and yield strength is often attributed to the material undergoing a thermal history, which, in turn, results in making its properties different, and generally worse, than those of the rest of the bulk material in the substrate [4].

Joining in the thixotropic state is considered to be an effective alternative joining method in which the material is processed when it is in a semisolid state between solidus and liquidus temperatures, that is, when the material shows thixotropic behaviour. Metal in a thixotropic state has many features such as high softness, good flowability, superb workability, and excellent join ability $[5,6]$. Therefore, the key potential advantage of the semisolid joining of alloys is that it can avoid many of the above-mentioned problems because of the fact that the solidification and heat transfer processes are basically different from those of typical welding. However, as reported by other studies, there are three methods that can be used to produce semisolid joining. The first method involves the use of a thixotropic metal as a filler applied into the joint groove for the purpose of joining materials [4]. The second method uses semisolid properties in combination with forming in a single process $[7,8]$. The third method 
TABLE 1: Chemical composition (wt\%) of AISI D2 tool steel and AISI 304 stainless steel by XRF technique.

\begin{tabular}{lccccccccc}
\hline & $\mathrm{C}$ & $\mathrm{Si}$ & $\mathrm{Mn}$ & $\mathrm{Cr}$ & $\mathrm{Ni}$ & $\mathrm{Mo}$ & $\mathrm{V}$ & $\mathrm{P}$ & $\mathrm{S}$ \\
\hline AISI D2 & 1.46 & 0.258 & 0.239 & 11.2 & 0.197 & 0.769 & 0.711 & - & - \\
AISI 304 & 0.08 & 1 & 2 & 18 & 8 & - & - & 0.04 & 0.03 \\
\hline
\end{tabular}

uses the properties of two different metals in their semisolid state [9]. It should be mentioned that these variants of semisolid joining technology are new processes that have been proposed fairly recently and thus far, researchers have not focused their attention on this particular area of work. Therefore, it is necessary to carry out fundamental investigations for the purpose of obtaining additional information about the general characteristics of this technology. In this study, thixojoining was applied to join AISI D2 cold-work tool steel to a 304 stainless steel. The joining process for this welded material was carried out by using a partial remelting method. The main aim of this paper is to provide globular joining structure in semisolid state. The study revealed that a new type of nonequilibrium diffusion interfacial structure was constructed at the interface of the two different types of steel.

\section{Experimental Procedure}

2.1. Materials. The investigated materials were AISI D2 (ENX160CrMo12-1) cold-work tool steel and AISI 304 stainless steel. The chemical composition of the starting materials is given in Table 1 .

2.2. JMatPro Simulation. JMatPro (Java-based material properties) is software that was developed to augment thermodynamic calculations by incorporating diverse theoretical material models and a properties database that allows quantitative calculation of the requisite material properties to be made within a larger software structure [10-12]. Here, JMatPro software is used to estimate solidus and liquidus temperatures as well as the liquid fraction profile within the semisolid zone for both metals.

2.3. Sample Preparation. The first step involved cutting the base metal (AISI D2) into samples with a size of $20 \times 10 \times$ $5 \mathrm{~mm}$ and cutting the insert metal (AISI 304 stainless steel) into samples with a diameter of $\varnothing 6 \times 5 \mathrm{~mm}$, as shown in Figure 1. In the second step each sample was immersed into a pickling solution with a concentration of $40 \mathrm{~mL} \mathrm{HCL} \mathrm{(37 \% )+}$ $60 \mathrm{~mL} \mathrm{H}_{2} \mathrm{O}$ for $5 \mathrm{~min}$ to get rid of rust and descale the metal to obtain a clean surface. In the third and final preparation step the sample was washed in acetone followed by rinsing with distilled water and then dried in air.

2.4. Direct Partial Remelting Experiment. Partial remelting is considered one of the most effective solid state routes to produce a nondendritic microstructure especially for high

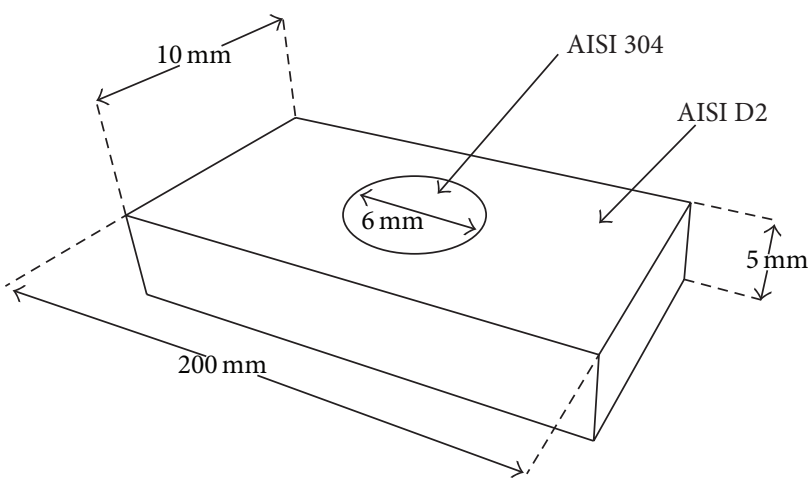

FIGURE 1: Diagram of the combination of the two different samples.

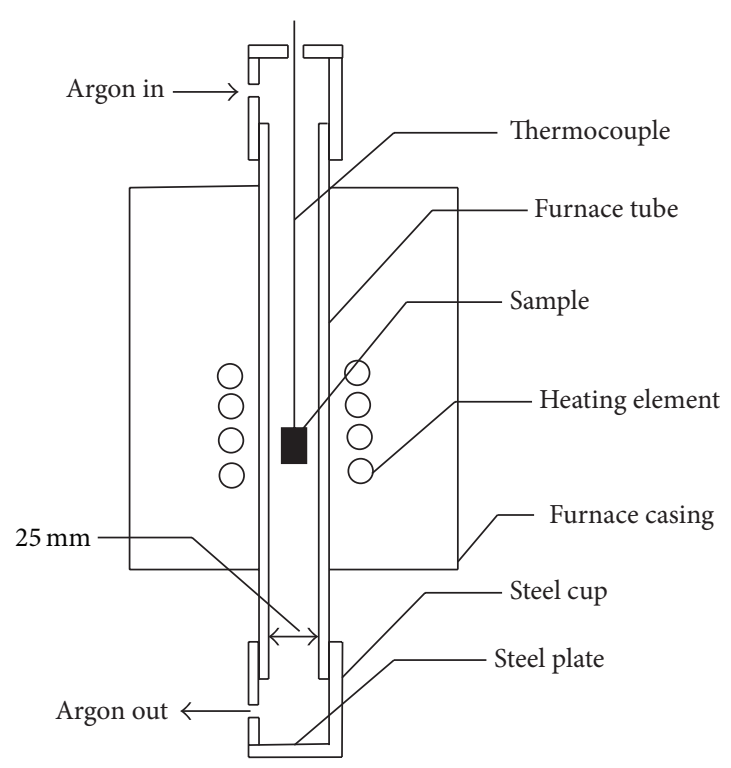

FIGURE 2: Schematic of the furnace setup for remelting experiment.

melting point metals when the metal is directly heated to a temperature between solidus and liquidus [13]. A direct partial remelting experiment was performed using a vertical, high temperature carbolite furnace with protective atmosphere of argon gas, as shown in Figure 2. When the furnace had reached the predefined temperature, the sample (base and insert) was lowered into the hottest place $\left(1320^{\circ} \mathrm{C}\right)$ of the furnace by using chromel wire and held there for $30 \mathrm{~min}$ after which it was subjected to air cooling to room temperature. This approach ensures a rapid heating of the sample which normally enables it to reach the predefined temperature in about 3 min, as shown in Figure 3. 


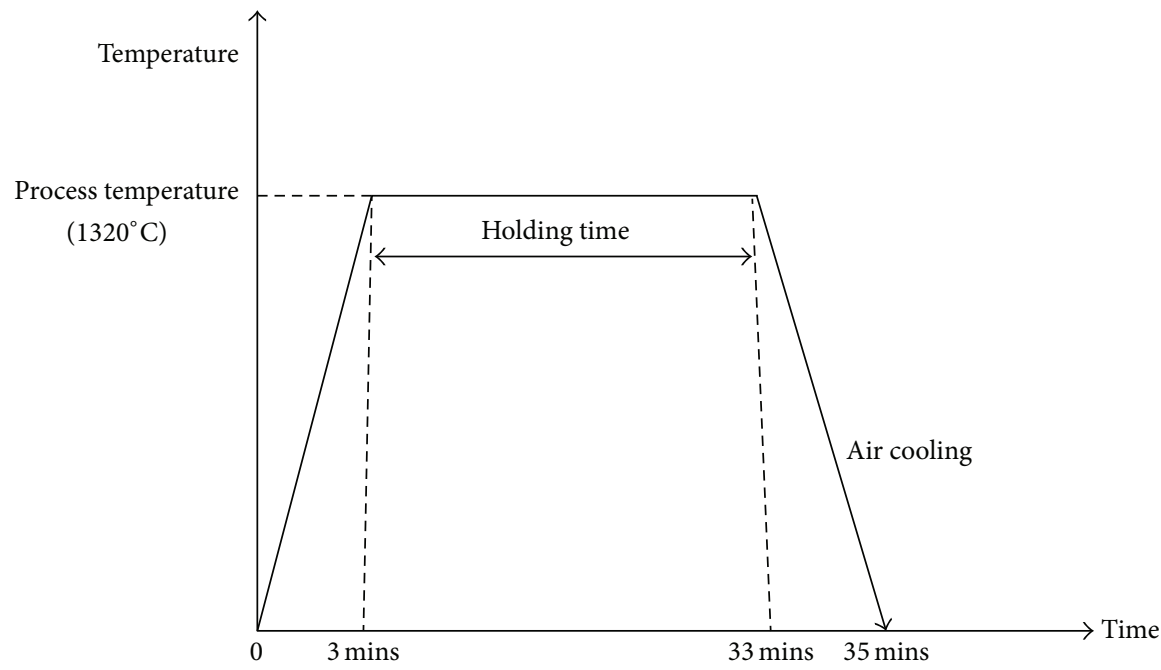

FIGURE 3: Temperature profile of sample inside vertical tube furnace.

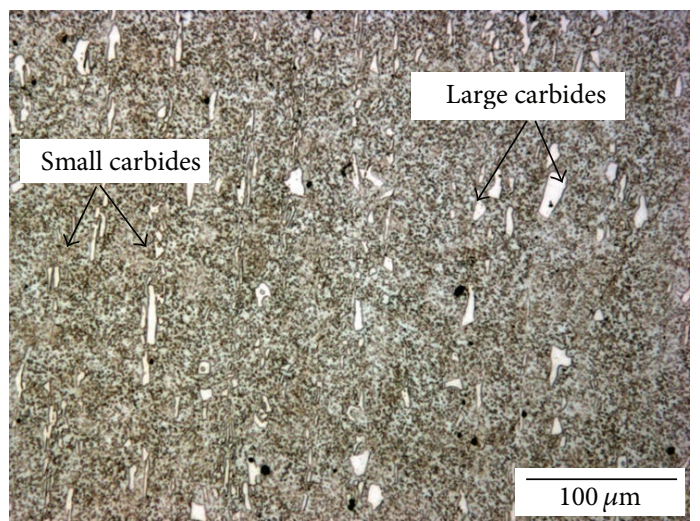

FIGURE 4: Optical micrographs of as-received AISI D2 tool steel.

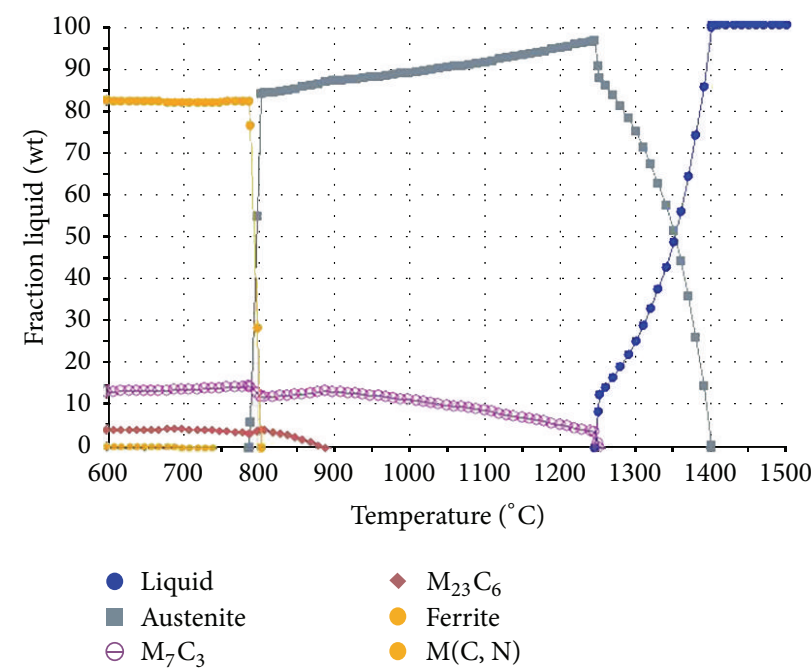

FIGURE 5: Phase equilibrium diagram of AISI D2 tool steel obtained from JMatPro simulation. 
2.5. Vickers Hardness Testing. Vickers hardness (HV) tests were performed on the transverse sections. The hardness measurement profiles were obtained through the sample's horizontal cross-section using a load of $2 \mathrm{~kg}$ applied for $15 \mathrm{sec}$.

2.6. Metallography and Image Analysis. Samples from the direct partial remelting experiments were carefully ground and polished to obtain mirror surfaces. Microstructural characterization was carried out by using a BX-51 Olympus optical microscope, a Hitachi S3400N scanning electron microscope (SEM), and an energy dispersive X-ray (EDX) equipped with energy (EDS dispersive spectroscopy). All samples were etched using Villela reagent ( $1 \mathrm{~g}$ picric acid, $5 \mathrm{~mL}$ hydrochloric acid, and $95 \mathrm{~mL}$ ethyl alcohol) to reveal their microstructures. Average grain sizes were calculated by using the mean lineal intercept method (after the ASTM E112-96 standard). The ideal grain size for semisolid forming should not exceed $100 \mu \mathrm{m}$ [14].

\section{Results and Discussion}

3.1. Starting Material. The as-received material contains surplus arrays of coarse large carbides with smaller carbides distributed homogeneously in a ferrite matrix parallel to the working direction, as shown in Figure 4 . This structure has been identified in annealed cold-work tool steel $[15,16]$ and thus confirms that soft annealing treatment had been carried out on the as-received material as described by the supplier. From Figure 5, the phase stability diagrams obtained from the JMatPro simulation for as-received AISI D2 show that, at room temperature, the carbides consisted of $\mathrm{M}_{7} \mathrm{C}_{3}, \mathrm{MC}$, and $\mathrm{M}_{23} \mathrm{C}_{6}$ type carbides, and these have been experimentally verified by Omar et al. [11].

3.2. Microstructure and Joint Quality. According to the graphs in Figure 6, the liquid fraction profile curve at $1320^{\circ} \mathrm{C}$ for AISI D2 was around 35\% while for AISI 304 stainless steel it was around $2.5 \%$. That is to say, there is a huge difference in the liquid fraction percentage at the same temperature between the base metal and the insert.

The microstructure of the welded joints revealed by the optical microscope is shown in Figure 7, while the microstructure of the welded joints observed by SEM is shown in Figure 8. The microstructure has three distinct features, the regions of which can be determined by the difference in colour and grain size. It can also be seen that there is a fine boundary between the base blank and insert with a smooth transition of the diffusion zone from one metal to the other. At the transition there is no evidence of microcracking or porosity, and the shape of the joint appears to be very smooth and not corrugated. A full penetration welded joint can also be clearly seen, as denoted by grain boundary migration and the observation of different colours in one grain. The grain size of AISI D2 is $85 \mu \mathrm{m}$, while the grains adjacent to the diffusion zone interface are around $140 \mu \mathrm{m}$. In addition, the base metal grains have changed through the semisolid process to form new columnar crystal growth
TABLE 2: EDX analysis and composition of the points marked in Figure 10 .

\begin{tabular}{lccc}
\hline Element & AISI D2 & Diffusion zone & AISI 304 \\
\hline $\mathrm{C}$ & 1.89 & 1.61 & 1.07 \\
$\mathrm{Si}$ & 0.21 & 0.28 & 0.36 \\
$\mathrm{P}$ & - & 0.033 & 0.037 \\
$\mathrm{Mo}$ & 0.79 & 0.39 & - \\
$\mathrm{S}$ & - & 0.04 & 0.05 \\
$\mathrm{~V}$ & 0.45 & 0.38 & - \\
$\mathrm{Cr}$ & 7.2 & 9.89 & 11.57 \\
$\mathrm{Mn}$ & 0.34 & 0.49 & 0.77 \\
$\mathrm{Ni}$ & 0.49 & 1.97 & 6.1 \\
$\mathrm{Fe}$ & 85.57 & 84.917 & 80.043 \\
\hline
\end{tabular}

near the convergence zone (diffusion zone). Nonequilibrium diffusion has occurred due to the difference in chemical composition of the two metals and the huge difference in the liquid fraction percentage of the two metals at the same temperature. As for the interfacial structure, both metals exhibit different reaction and diffusion abilities between solidus and liquidus whereby the base metal is connected to the insert metal along the bonding boundary to achieve what appears to be a perfect joining at the interfaces of both metals.

Scanning electron microscopy and EDX analysis combined with EDS analysis were carried out in order to scrutinize the phases that occurred during the joining process at the interface region. The line scan analysis was performed along the marked line as shown in Figure 9. From this figure it is observed that nickel and chromium diffuse towards the AISI D2 tool steel from the AISI 304 stainless steel and the diffusion of iron and carbon from tool steel side towards stainless steel. Figure 10 illustrates the EDX analysis results from the SEM image taken from points 1,2, and 3 representing the AISI-D2, the diffusion zone, and 304 stainless steel in the AISI D2-AISI 304 joint, respectively, while distribution of elements within the determined location is shown in Table 2. It can be clearly seen that elements $\mathrm{P}$ and $\mathrm{S}$ begin to transfer from the AISI 304 side in the interface region and grain boundaries, while $\mathrm{Mo}, \mathrm{V}$, and $\mathrm{Cu}$ of AISI D2 side diffuse into the convergence zone. The EDS results confirm the formation of congregation compounds of these two different metals. The formation of the new phase compounds has a tendency to depend on the difference in the chemical composition of the two metals, which leads to the diffusion of different elements through the interface region (diffusion zone).

3.3. Vickers Hardness Testing. The variation in the hardness values in the transverse cross-section of the joint is shown in Figure 11. It can be seen that the hardness values obtained from the points in the base metal are much higher than those in the insert, while in the diffusion zone the hardness value is higher than that in the insert but lower than that in the base metal. These differences are probably due to the diffusion of the elements from both metals at the interface zone of the 


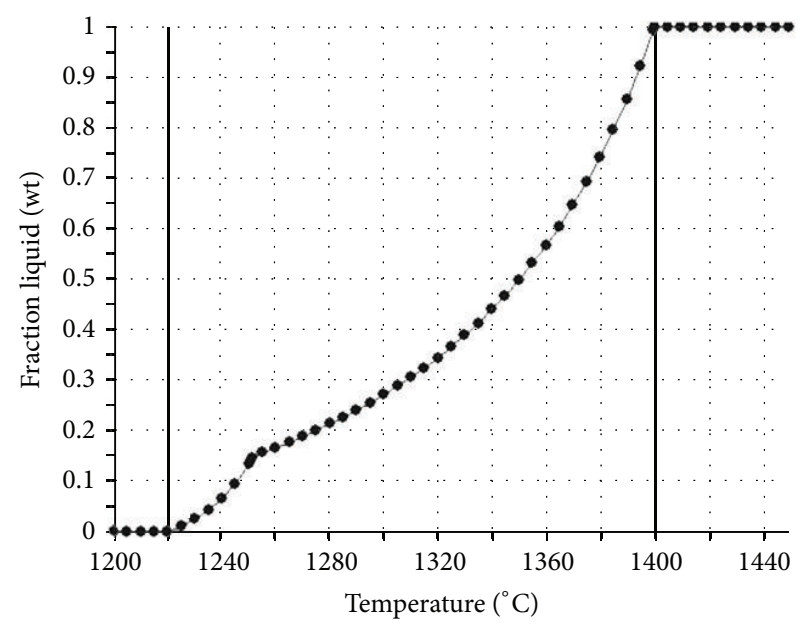

(a)

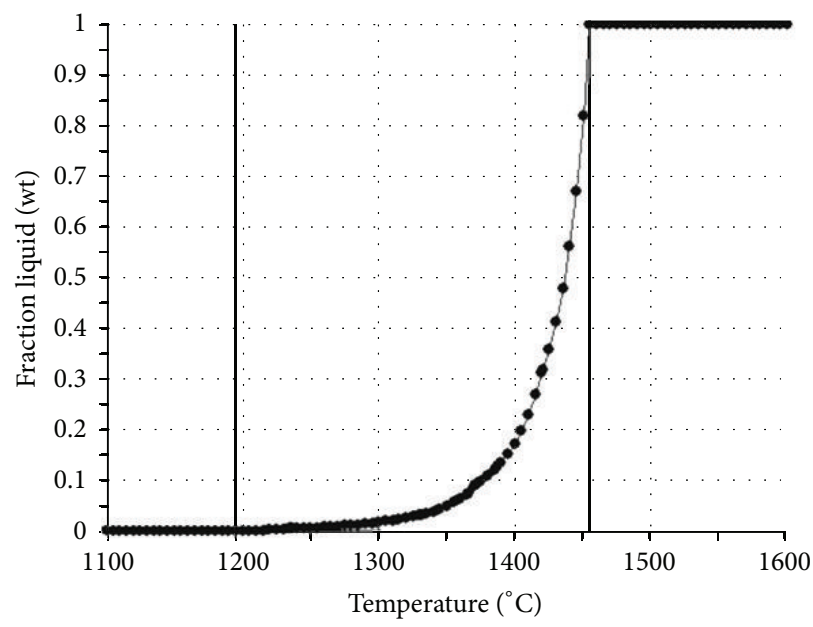

(b)

FIGURE 6: Liquid fraction profile for chemical composition of (a) AISI D2 tool steel and (b) AISI 304 stainless steel obtained from JMatPro simulation.
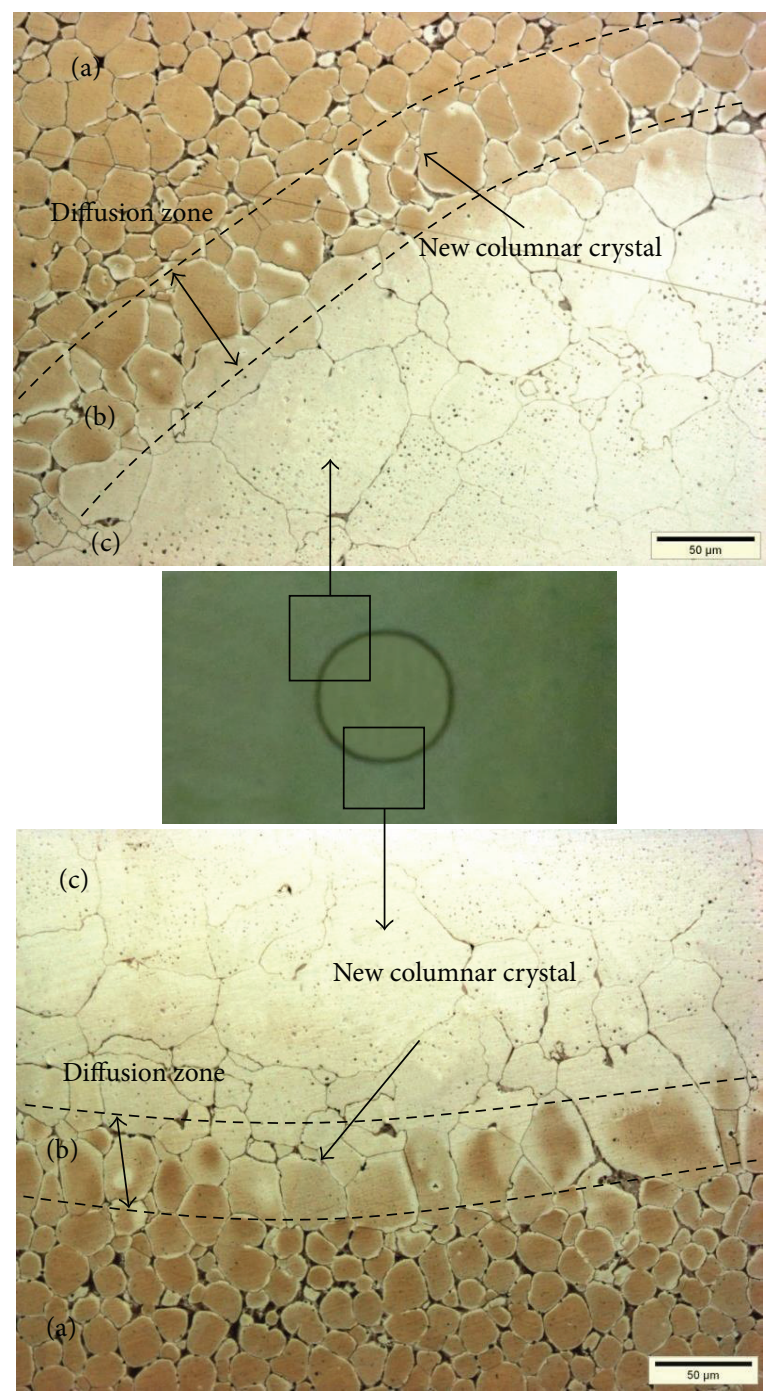

FIgURE 7: Microstructures of thixojoint of (a) AISI D2, (b) diffusion zone, and (c) AISI 304 after application of $1320^{\circ} \mathrm{C}$ for 30 minutes. 


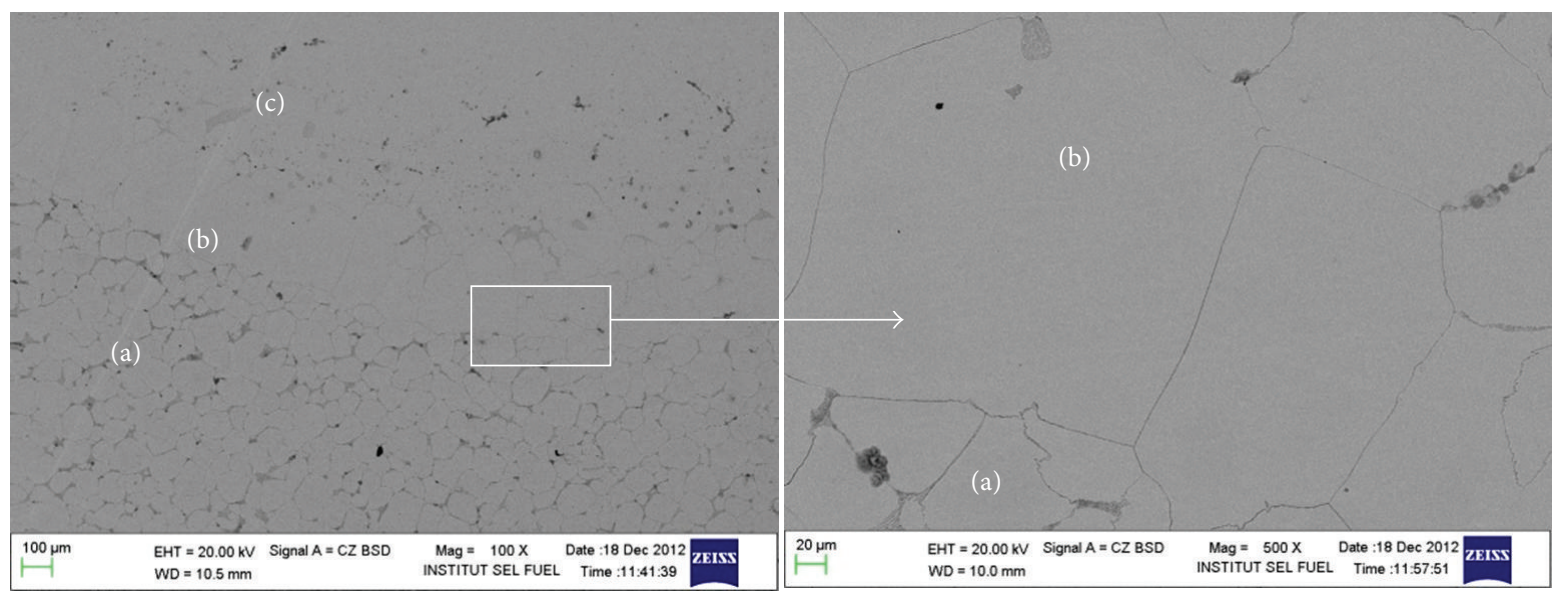

FIGURE 8: Image of microstructures of (a) AISI D2, (b) diffusion zone, and (c) AISI 304.

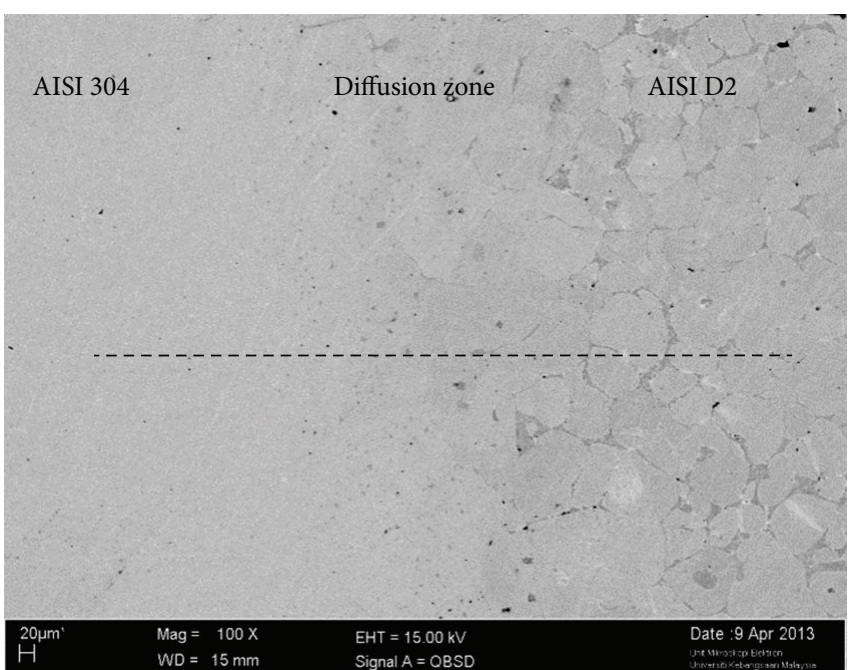

(a)

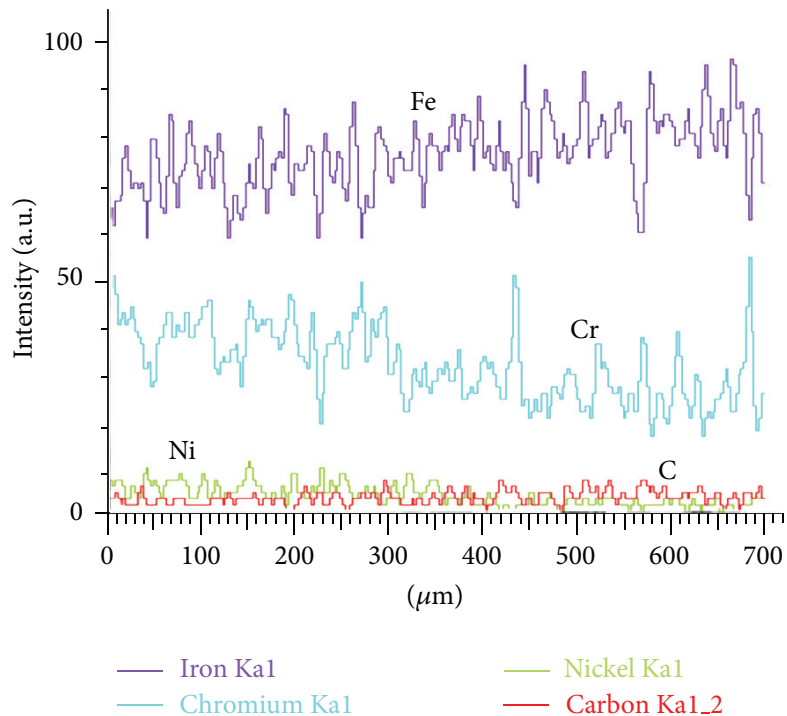

(b)

FIGURE 9: Elemental distribution across the joint centre in AISI D2-AISI 304.

joint. Then, there is a decrease towards the side of the insert part on the horizontal distance of the joints, as shown in Figure 11. This hardness distribution data for the transverse cross-section of joints can be attributed to the fine transition structure between the base metal and the insert as well as to the natural diffusion between these different metals. It can be seen that the hardness of the cross-section area is almost uniform throughout the whole length of the sample.

\section{Conclusion}

A new type of thixojoining process for AISI D2 with AISI 304 stainless steel using a partial remelting method was proposed. This process is not based on conventional methods (adhesive, nails, and screws) but can produce homogeneous properties with high surface quality and avoids the creation of a dendritic microstructure at the join zone. In addition, this technique has two key advantages when compared with conventional joining methods, namely, the possibility of producing functional components with multiple materials and minimizing the defects associated with conventional welding processes. In addition, by using this type of thixojoining process, there is no need for force or complex equipment when compared to conventional welding processes such as friction welding. Based on the results of the experiments conducted in this study, the use of this thixojoining technique can create a fine interfacial diffusion along the bonding boundary between the base blank and insert. Furthermore, a smooth transition from one metal to the other is achieved through the formation of new columnar crystal growth near the convergence zone. The transition occurs without evidence of microcracking or porosity. 


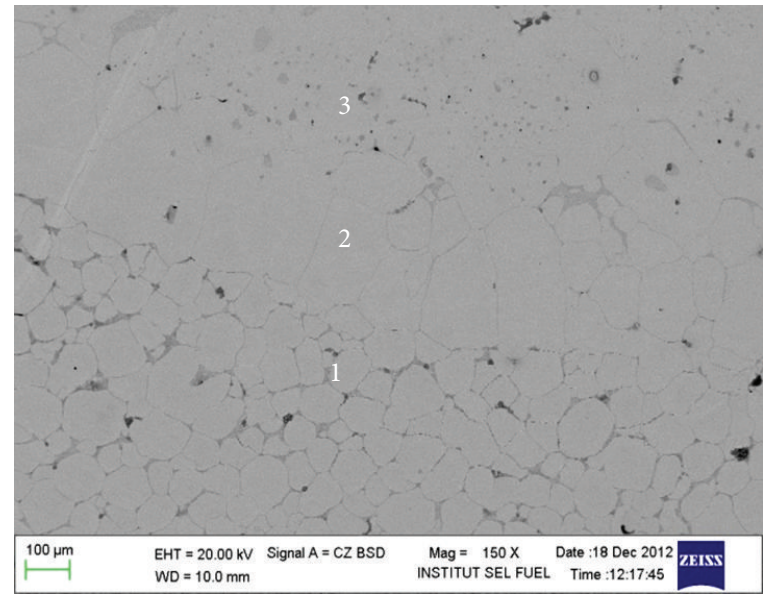

(a)

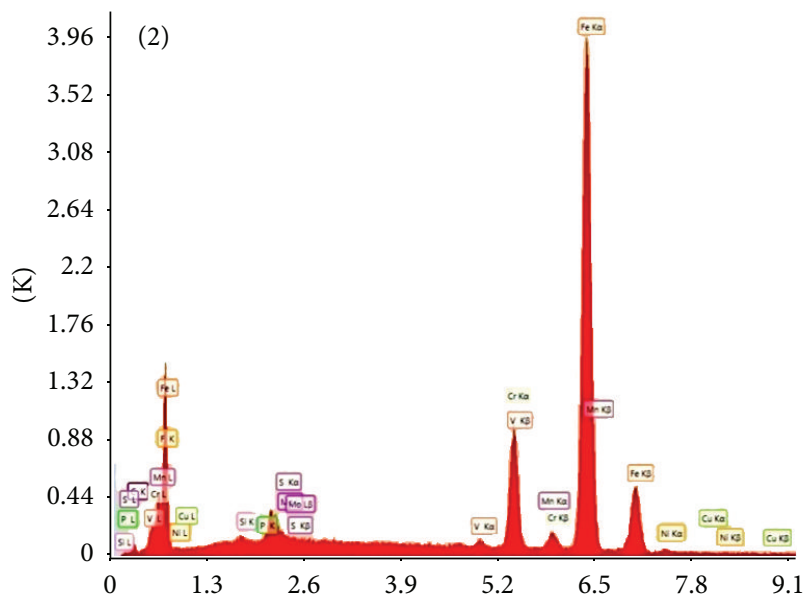

(c)

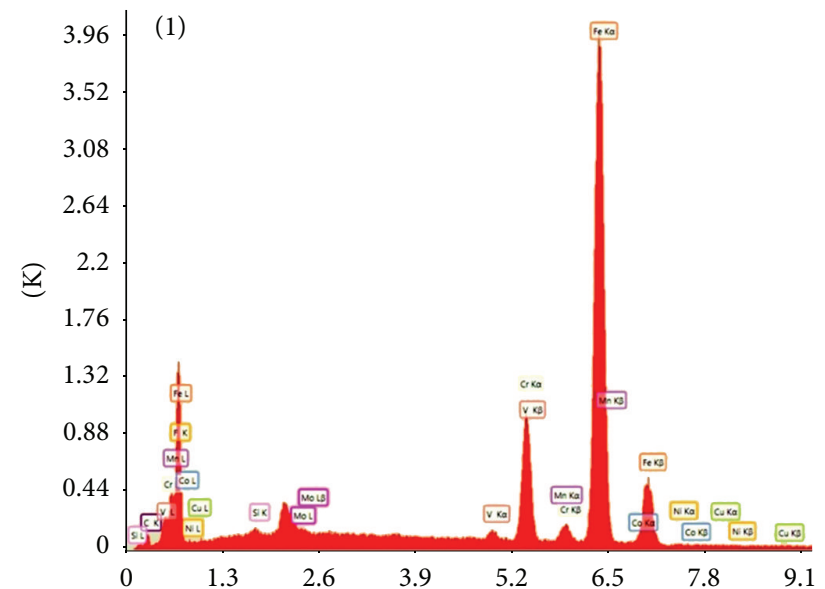

(b)

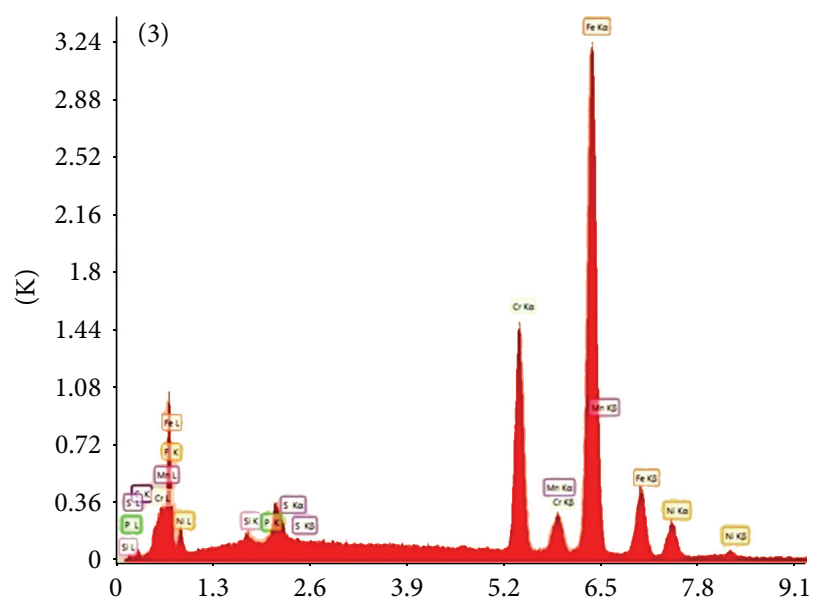

(d)

FIGURE 10: SEM micrograph of joining compound and EDX analysis at (point 1) AISI D2, (point 2) diffusion zone, and (point 3 ) 304 stainless steel.

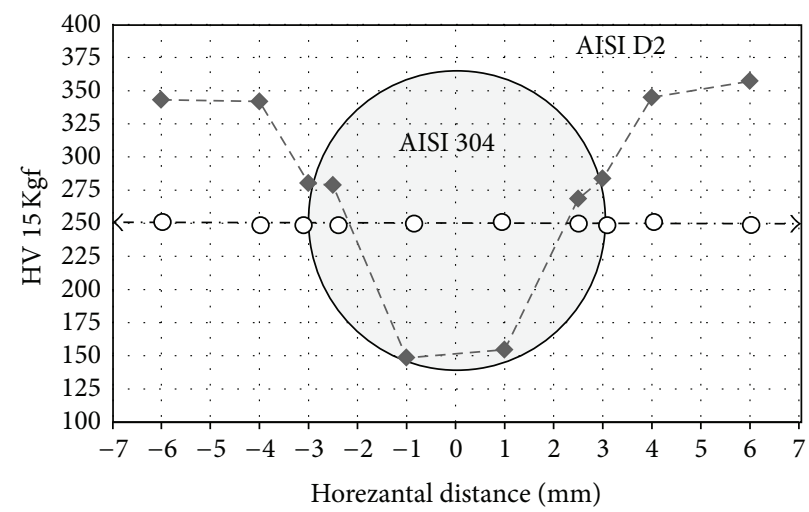

FIGURE 11: Vickers hardness profile. 


\section{Acknowledgments}

The authors would like to thank Universiti Kebangsaan Malaysia (UKM) and the Ministry of Science, Technology and Innovation (MOSTI), Malaysia for the financial support under research Grants GUP-2012-040 and AP-2012-014.

\section{References}

[1] G. Govender, L. Ivanchev, H. Burger, R. Knutsen, and G. Kunene, "Weldability of SSM rheo processed aluminum alloy A356," Solid State Phenomena, vol. 141-143, pp. 773-778, 2008.

[2] S. Zhang, X. Guo, and G. Ma, "Investigation of semisolid welding characteristics of 2A14 aluminum alloy," Advanced Materials Research, vol. 154-155, pp. 639-642, 2011.

[3] A. Narimannezhad, H. Aashuri, A. H. Kokabi, A. Khosravani, M. Kiani, and A. Foroughi, "Semisolid joining of zinc AG40A alloy by partial remelting and mechanical stirring," Solid State Phenomena, vol. 141-143, pp. 225-230, 2008.

[4] P. F. Mendez, C. S. Rice, and S. B. Brown, "Joining using semisolid metals," Welding Journal, vol. 81, no. 9, pp. 181-187, 2002.

[5] M. Kiuchi, J. Yanagimoto, and S. Sugiyama, "Mashy-state joining, a new process for joining materials together," in Proceedings of the 5th International Conference on Semi-Solid Processing of Alloys and Composites, pp. 123-130, Golden, Colo, USA, 1998.

[6] M. Kiuchi, J. Yanagimoto, and S. Sugiyama, "Application of mushy/semi-solid joining part-2," in Proceedings of the 7th International Conference on Semi-Solid Processing of Alloys and Composites, pp. 707-712, Tsukuba, Japan, 2002.

[7] R. Baadjou, H. Shimahara, and G. Hirt, "Automated semi-solid forging of steel components by means of thixojoining," Solid State Phenomena, vol. 116-117, pp. 383-386, 2006.

[8] R. Kopp, J. Kallweit, T. Moller, and I. Seidl, "Forming and joining of commercial steel grades in the semi-solid state," Journal of Materials Processing Technology, vol. 130-131, pp. 562-568, 2002.

[9] M. N. Mohammed, M. Z. Omar, M. S. Salleh, M. A. Zailani, and K. S. Alhawari, "Joining two metals via partial remelting method," Journal of Asian Scientific Research, vol. 2, pp. 724-730, 2012.

[10] N. Saunders, Z. Guo, X. Li, A. P. Miodownik, and J. P. Schillé, "Using JMatPro to model materials properties and behavior," JOM, vol. 55, no. 12, pp. 60-65, 2003.

[11] M. Z. Omar, A. Alfan, J. Syarif, and H. V. Atkinson, "Microstructural investigations of XW-42 and M2 tool steels in semi-solid zones via direct partial remelting route," Journal of Materials Science, vol. 46, no. 24, pp. 7696-7705, 2011.

[12] M. S. Salleh, M. Z. Omar, J. Syarif, and M. N. Mohammed, "Thermodynamic modelling on the mutual effect of copper, manganese and iron addition in Al-Si-Cu for semisolid processing," Journal of Asian Scientific Research, vol. 2, pp. 614-619, 2012.

[13] M. Z. Omar, H. V. Atkinson, and P. Kapranos, "Thixotropy in semisolid steel slurries under rapid compression," Metallurgical and Materials Transactions A, vol. 42, no. 9, pp. 2807-2819, 2011.

[14] G. Hirt, L. Khizhnyakova, R. Baadjou, F. Knauf, and R. Kopp, "Semi-solid forming of aluminium and steel-introduction and overview," in Thixoforming-Semi-Solid Metal Processing, G. Hirt and R. Kopp, Eds., pp. 1-27, Willey-VCH GmbH, Weinheim, Germany, 2009.
[15] G. A. Roberts, J. C. Hamaker Jr., and A. R. Johnson, Tool Steels, American Society for Metals, Materials Park, Ohio, USA, 3rd edition, 1971.

[16] A. Alfan, M. Z. Omar, J. Syarif, and Z. Sajuri, "Direct partial remelting of XW-42 steel in semi-solid zone," Journal of Applied Sciences, vol. 10, no. 13, pp. 1255-1262, 2010. 

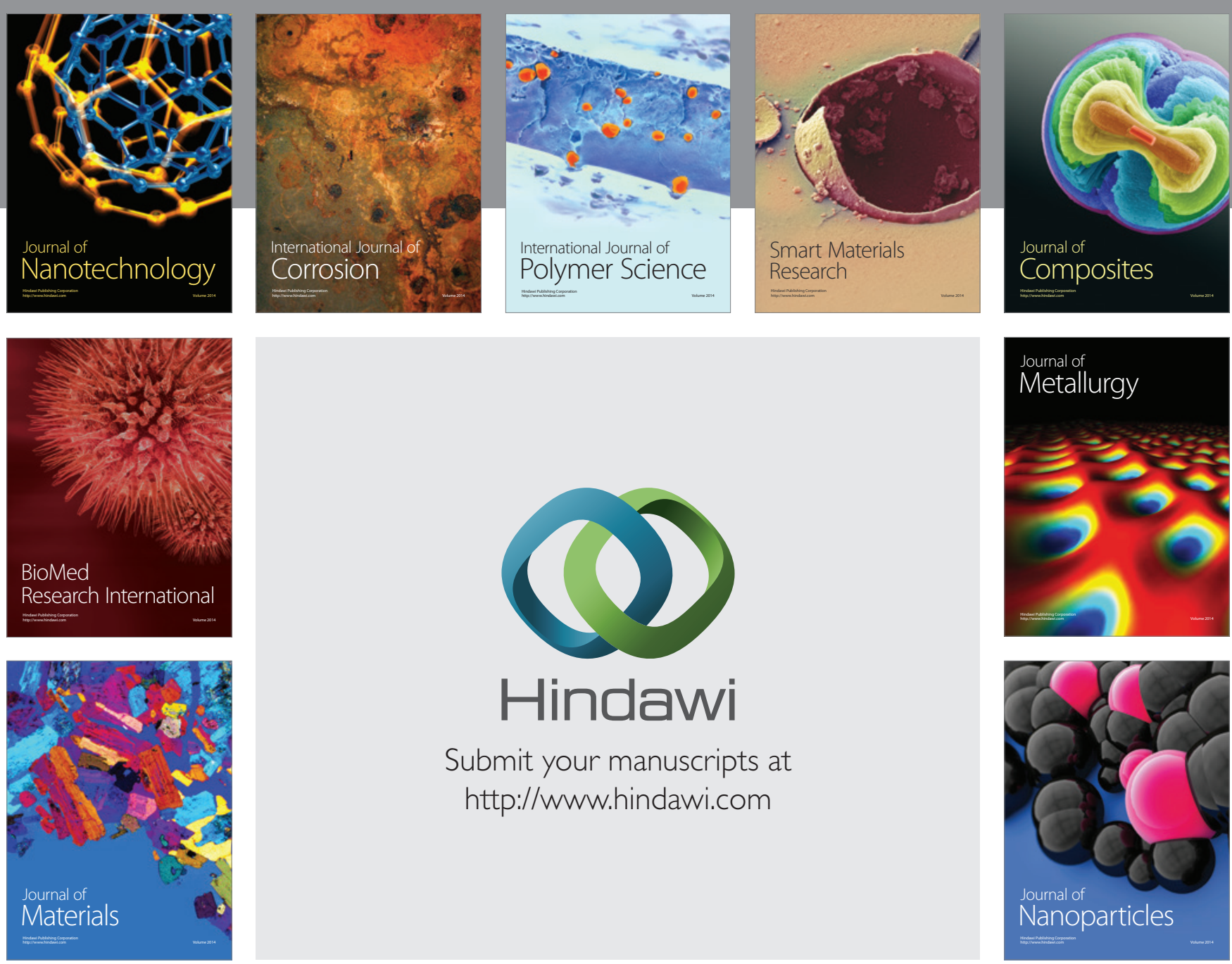

Submit your manuscripts at http://www.hindawi.com
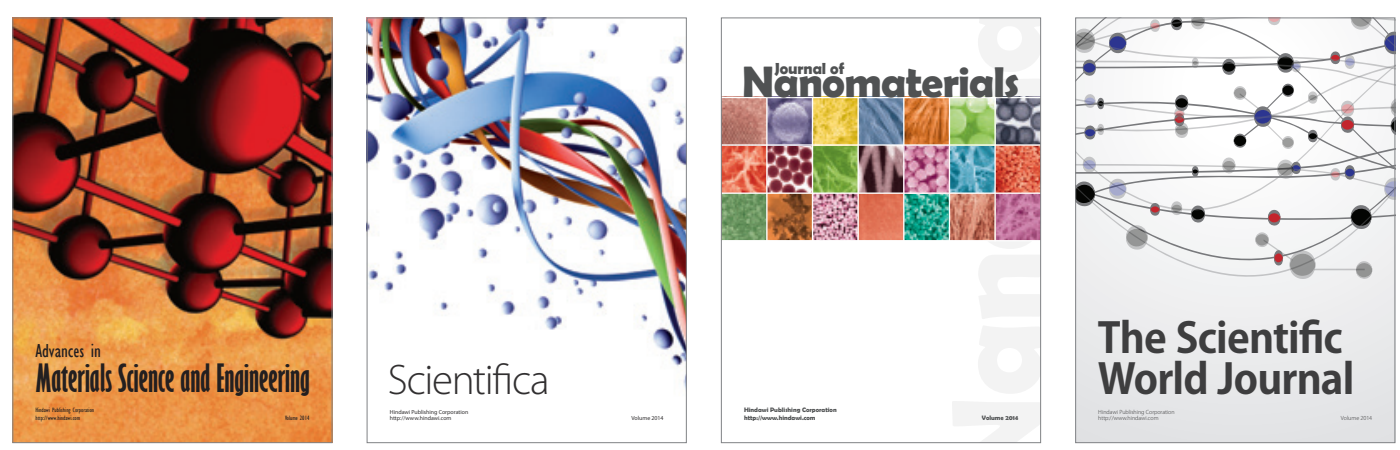

\section{The Scientific World Journal}
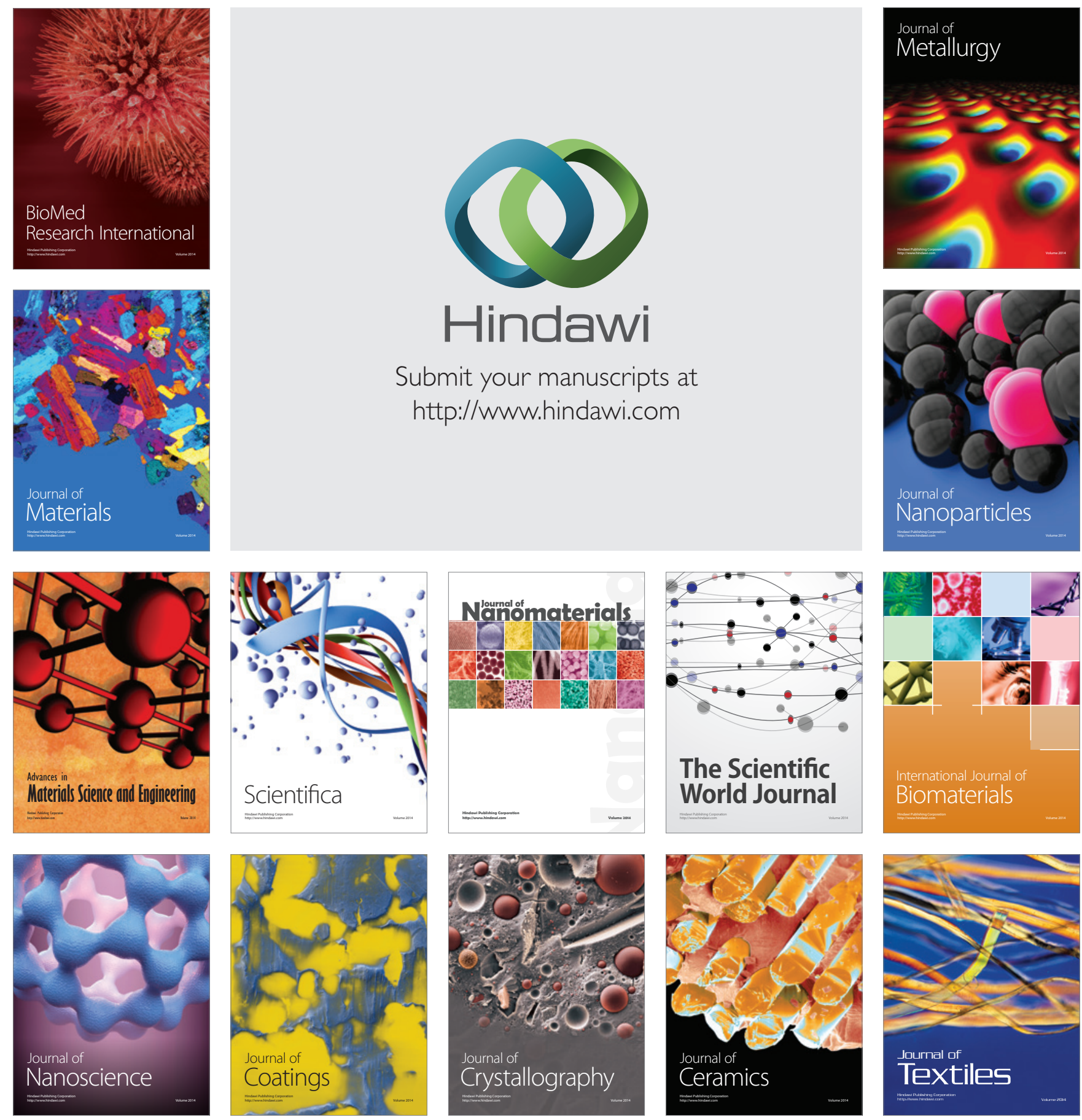